\title{
Prey selection by thaidid gastropods: some observational and experimental field tests of foraging models
}

\author{
A. Richard Palmer * \\ Department of Zoology, University of Washington, Seattle, Washington 98195, USA
}

\begin{abstract}
Summary. Field observations and experiments revealed that predatory intertidal gastropods of the genus Thais (or $\mathrm{Nu}$ cella) were able both to recognize the expected food value of encountered prey (expected energy or growth potential gained per unit handling time) and to monitor their average yield over time (average energy or growth potential gained per unit foraging time). They appeared to discriminate not only among prey species, but also among different sized individuals of the same prey species. The evidence supporting these interpretations included: 1) field observations of snails feeding preferentially on prey types of higher expected food value even though lower value prey types were available and abundant, 2) a very limited number of direct underwater observations of foraging snails rejecting encountered items that were either of lower expected value than the item finally eaten or not measurably different from it, and 3) field (='arena') experiments in which both average yield, and the distribution and abundance of potential prey were controlled: snails conditioned at a high average yield fed preferentially on high value items, while those animals conditioned at a low yield consumed prey in the proportions that they were encountered. These behaviors are all consistent with a prey-selection decision motivated by energetic considerations. Further, the field experiments indicated that these predatory gastropods could select items from a diverse array of prey so as to maximize growth in their natural environment. The behaviors were not consistent with three alternative foraging hypotheses: non-selective foraging, frequency-dependent foraging on prey types (here, sizes of particular prey species), and frequency-dependent foraging on prey species. Deviations from some of the quantitative predictions of optimal foraging theory appeared related to learning and risk.
\end{abstract}

\section{Introduction}

Factors that influence a consumer's choice of food have received considerable recent attention (see reviews by Hughes 1980; Schluter 1981). A recurring premise is that consumers should select food in such a way as to maximize their net rate of energy intake, although other factors may also be important. The theoretical problem of which food

* Present address: Department of Zoology, University of Alberta, Edmonton, Alberta, T6G 2E9, and Bamfield Marine Station, Bamfield, British Columbia, V0R 1B0, Canada items to select from an array of types that may vary widely in energetic value, ease of consumption or availability has been addressed by a number of authors (Charnov 1976; Estabrook and Dunham 1976; Hughes 1979; Pulliam 1974, 1975; Schoener 1971; Werner and Hall 1974). However, in spite of numerous observational (Belovsky 1978; GossCustard 1977; Ostfeld 1982; Werner and Mittlebach 1981) and experimental (Draulans 1982; Ebersole and Wilson 1980; Elner and Hughes 1978; Krebs et al. 1977; Lacher et al. 1982; Werner and Hall 1974) tests of the optimal diet model, some questions still remain (in particular, see Schluter 1981 for a critical review): 1) to what extent are these models useful in predicting consumer behavior quantitatively where several substantially different kinds of prey (e.g. prey species) may be present, and 2) how accurately do these models predict the quantitative pattern of prey selection in the field?

To address these questions, I examined prey selection by three marine gastropod species of the genus Thais (or Nucella): T. lamellosa, $T$. canaliculata and T. emarginata. All three species are common inhabitants of rocky intertidal shores from Alaska to California (Abbott 1974; Ricketts et al. 1968) and prey primarily on barnacles (Balanus glandula, Semibalanus cariosus, Chthamalus dalli, and occasionally Pollicipes polymerus) and mussels (Mytilus edulis and to a much lesser extent $M$. californianus) (Palmer 1980). This system of predators and prey is well suited to a quantitative study of foraging behavior. Predators may be marked individually for unambiguous identification; they are slow moving (Miller 1974) and hence easy to follow while foraging; they are not disturbed by close observation; and the environment in which they live is largely two-dimensional. Because they are non-visual predators relying entirely on chemosensory or tactile cues and because their principal prey are all effectively sessile, both acceptance and rejection of individual food items may be assessed with a high degree of certainty. In addition, both the prey and the predators are readily manipulable; snails may be offered restricted feeding regimes, and the distribution and abundance of individual prey items can be controlled experimentally.

\section{Methods}

\section{Measurement of food value and size}

To obtain measures of food value under conditions of normal tidal exposure, I grew Thais from three size classes of each species on pure diets of their principal prey species 
Balanus glandula, Semibalanus cariosus, and Mytilus edulis in cages mounted in the intertidal. Individuals of each prey species were divided into four discrete size classes spanning nearly the full size range of each species and maintained in sufficient abundance so search times were minimal and uneaten prey were always available to the predators. Growth of individually marked snails (four/prey type) was measured as percent body weight gain (Palmer 1982) over a 30 day interval at each of two tidal heights for each Thais species. Additional experimental details and results are published elsewhere (Palmer 1983). In the subsequent analyses, the expected food values (their average potential for promoting growth) of eaten and available prey were linearly interpolated when necessary between the expected values of adjacent size classes. Prey outside the largest or smallest terminal size classes were assigned the food value of the nearest terminal size class. Throughout, numerical values of expected food value correspond to total percent change in body weight over 30 days when grown on pure diets of a particular prey type.

Sizes of snails are reported as shell lengths (apex to tip of siphonal canal), mussel sizes are shell lengths (maximum dimension parallel to the long, ventral margin of the shell), and barnacle sizes are opercular diameters (rostrocarinal diameter inside the aperture). All size measurements were recorded to $0.1 \mathrm{~mm}$ with vernier calipers. MLLW

Cited tidal heights refer to height above US datum,

\section{Field observations}

To assess prey choice by unmanipulated snails in the field, two types of observations were made. The first ('low-tide observations') were obtained from individual Thais emarginata observed feeding at low tide and included measurements of: 1) predator size, 2) size and species of the prey item being eaten (in all cases an extended proboscis or evidence of drilling was required to verify feeding), 3) approximate tidal height of the location [heights of observations were estimated as being at one of the two heights used when growing species in the controlled feeding experiments $(+2.0 \mathrm{ft},+0.7 \mathrm{~m}$ or $+4.0 \mathrm{ft},+1.3 \mathrm{~m}$; Palmer 1983)], and 4) size and species of all prey within $50-70 \mathrm{~mm}$ of the feeding snail (=available prey). The maximum distance to which available prey were recorded was arbitrary and chosen only to provide an approximation of the relative abundances of each prey type encountered before the snail initiated feeding. Because of small-scale spatial heterogeneity in the distribution of prey, a larger sample area might not have provided an accurate reflection of the proportions of prey encountered immediately prior to feeding.

The second type of observation ('high-tide observations') was obtained by recording the prey items encountered and rejected by foraging snails at high tide with the aid of SCUBA. Individual $T$. lamellosa and $T$. emarginata were numbered (Palmer 1980), measured for shell length and released where captured on Point George, Shaw Island, WA (USA). Two weeks after release, animals were observed after they were immersed by a rising tide. For each snail, the time of encounter, the species' identity and the size of each prey item encountered was recorded. Only items contacted physically and bypassed were scored as 'rejected'. Items attacked were verified as eaten on the following low tide.

\section{Cage and arena experiments}

To assess the impact of immediate past feeding history on subsequent prey selection, two sets of experiments were conducted in which average yields were manipulated and prey choice monitored: 1) in cages attached to pilings at two tidal heights ('cage experiments') and 2) in foraging arenas on a rocky shore ('arena experiments'). In the cage experiments, snails were exposed to different yields ('conditioned') in $3 \mathrm{~mm}$ VEXAR mesh cages mounted on pilings under the Friday Harbor Laboratories (FHL) dock, Friday Harbor, WA (USA, $48^{\circ} 33^{\prime} \mathrm{N}, 123^{\circ} 01^{\prime} \mathrm{W}$ ). The cages were deployed at tidal heights within the vertical range of each predator species as indicated in Tables 1-3. Five numbered snails of a restricted size range were placed with appropriate prey (a narrow size range of a single prey species) in each of two replicate cages at each tidal height, and allowed to feed ad libitum at these different yields for 26 days (Aug. 8 to Sept. 3, 1978). Two- to three-hundred barnacles were provided per cage on small stones; 40-50 mussels (M. edulis) were introduced into the cage one week before before loading the snails to allow them at attach. Two weeks after introducing the snails, the prey were checked and replenished where necessary, thus a surfeit of prey was always available.

Before the end of the conditioning interval, $15 \times 120 \mathrm{~cm}$ observation cages (Fig. 1) were constructed to permit prey choice to be monitored. Equal numbers of four prey types were provided in each observation cage ( 24 items per prey type per cage; details in Fig. 1 and Tables 3-5). Individual Mytilus edulis of appropriate sizes were collected from the FHL dock. Small B. glandula (approx. $2 \mathrm{~mm}$ opercular diameter) were collected on $M$. edulis valves from Sinclair Inlet (Bremerton, WA, USA; $47^{\circ} 32^{\prime} \mathrm{N}, 122^{\circ} 40^{\prime} \mathrm{W}$ ), the mussel flesh was removed from the valves and then they were broken into small fragments with three to six barnacles per shell fragment. Individual medium-sized (4-5 mm) and large $(>8 \mathrm{~mm})$ B. glandula were obtained from Wescott Bay (San Juan Island, WA, USA; $48^{\circ} 36^{\prime} \mathrm{N}, 123^{\circ} 08^{\prime} \mathrm{W}$ ) as barnacles that had settled and grown upon others: by dislodging the basal barnacles, the epizooic ones could be collected without damage. All prey items were glued individually into the cages in a uniform distribution $(4 \times 5 \mathrm{~cm}$ grid) with underwater epoxy (Sea Goin' 'Poxy Putty, Permalite Plastics, Newport Beach, CA). The grid on the bottom of the cage (Fig. 1) also permitted coordinates of feeding individuals to be noted both for later verification of consumption and ensured that snails found at the same item on two or more successive inspections were not counted as separate feeding observations.

At the end of the conditioning interval, prey selection was monitored in observation cages at the same tidal height at which the snails had been conditioned. Five $T$. lamellosa and five $T$. canaliculata from each conditioning treatment were introduced into a single observation cage ( 25 snails per species at the low level and 20 per species at the mid level). Two replicate observation cages were established at both the low $(0.0 \mathrm{ft}, 0.0 \mathrm{~m})$ and the mid $(+2.0 \mathrm{ft},+0.7 \mathrm{~m})$ level. Five $T$. emarginata from each treatment condition were placed in paired replicate cages (25 snails per cage) and the cages were then depolyed at the high level $(+4.0 \mathrm{ft}$, $+1.3 \mathrm{~m}$ ). Prey choice in all cages was monitored twice daily for twelve days, once at low tide and once at high tide with the aid of SCUBA. After six days all drilled or con- 

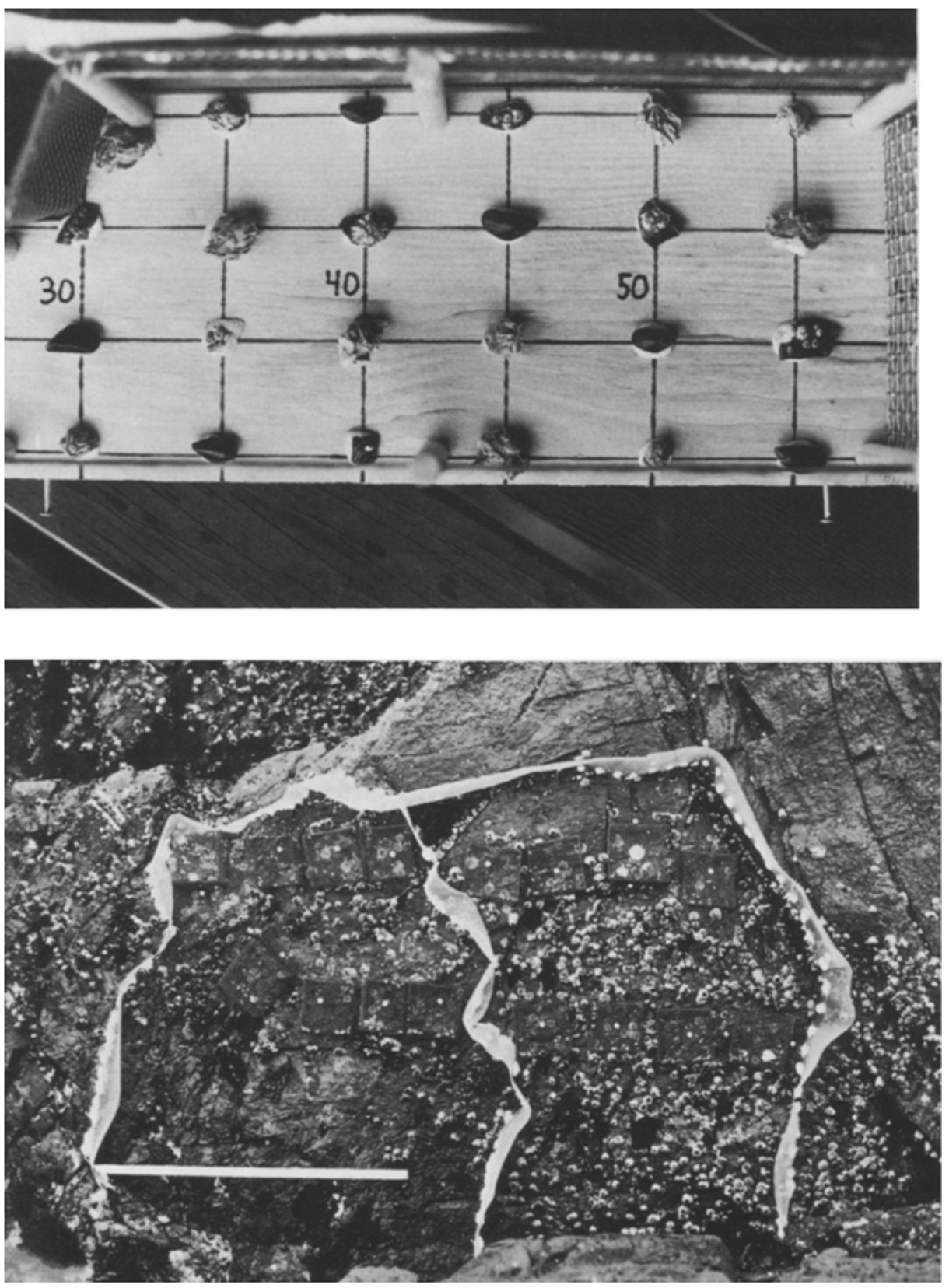

iig. 1. Close-up of a portion of the nside of an observation cage from he cage experiments, illustrating he uniform distribution of four rey types on a $4 \times 5 \mathrm{~cm}$ grid. From op to bottom on the $40 \mathrm{~cm}$ line: mall Mytilus edulis, medium 3 alanus glandula, large B. glandula, nd small $B$. glandula on a

ragment of mussel valve. The sides if the cage were of fine-mesh IEXAR (R) plastic screen and the op was of clear MYLAR (R)
Fig. 2. Conditioning cages and foraging arenas for the arena experiments conducted on Deadman Island, San Juan Islands, Washington (USA). A meter stick is present for scale sumed items were removed from the observation cages and replaced with fresh prey; snails were not disturbed while feeding during these periods.

A second set of experiments ('arena' experiments) assessing the impact of immediate past feeding history on prey selection, was conducted on the shore of Deadman Island, WA (USA : $48^{\circ} 28^{\prime} \mathrm{N}, 122^{\circ} 57^{\prime} \mathrm{W}$ ) and was repeated twice in 1978 [first series: Thais conditioned from July 23 to Aug. 16 (24 days), prey selection monitored from Aug. 17 to Aug. 22 (5 days); and second series: conditioned from Aug. 30 to Sept. 25 (26 days), monitored from Sept. 25 to Oct. 2 (8 days)]. Each series consisted of two replicates for each of four treatment groups (see below). Each replicate was situated within a restricted 'arena' approximately $1.5 \mathrm{~m}^{2}$ in area $(1.0 \times 1.5 \mathrm{~m}$; Fig. 2) surrounded by approximately $10 \mathrm{~cm}$ wide strips of fine-mesh $(3 \mathrm{~mm}$ ) VEXAR (Dupont) plastic screen bonded to the rock with Sea Goin'
'Poxy Putty. To expedite monitoring, a $10 \mathrm{~cm}$ square gridwork was made in the foraging arenas using small dabs of "Poxy Putty pressed into the rock as markers.

In these arena experiments, the four yield-treatments included: 1) starved (control), 2) small (approx. $2 \mathrm{~mm}$ ) $B$. glandula (low yield, barnacle), 3) $30 \mathrm{~mm}$ Mytilus edulis (low yield, mussel), and 4) intermediate sized (4-6 mm) Balanus glandula (high yield, barnacle). Prey were provided in $15 \times 15 \times 5 \mathrm{~cm}$ cages of $5 \mathrm{~mm}$ mesh galvanized steel, hardware cloth (Fig. 2) that were attached in the intertidal with stainless stell screws and masonry anchors at $+2.5 \mathrm{ft}$ $(+0.8 \mathrm{~m})$. Only one type of prey was provided per cage. Intermediate sized $B$. glandula were supplied on small stones cemented to the bottom inside the cages using Sea Goin' 'Poxy Putty. Mussels in the appropriate size range were placed in the cages and allowed to strengthen their attachment for at least one week before introducing the snails. 


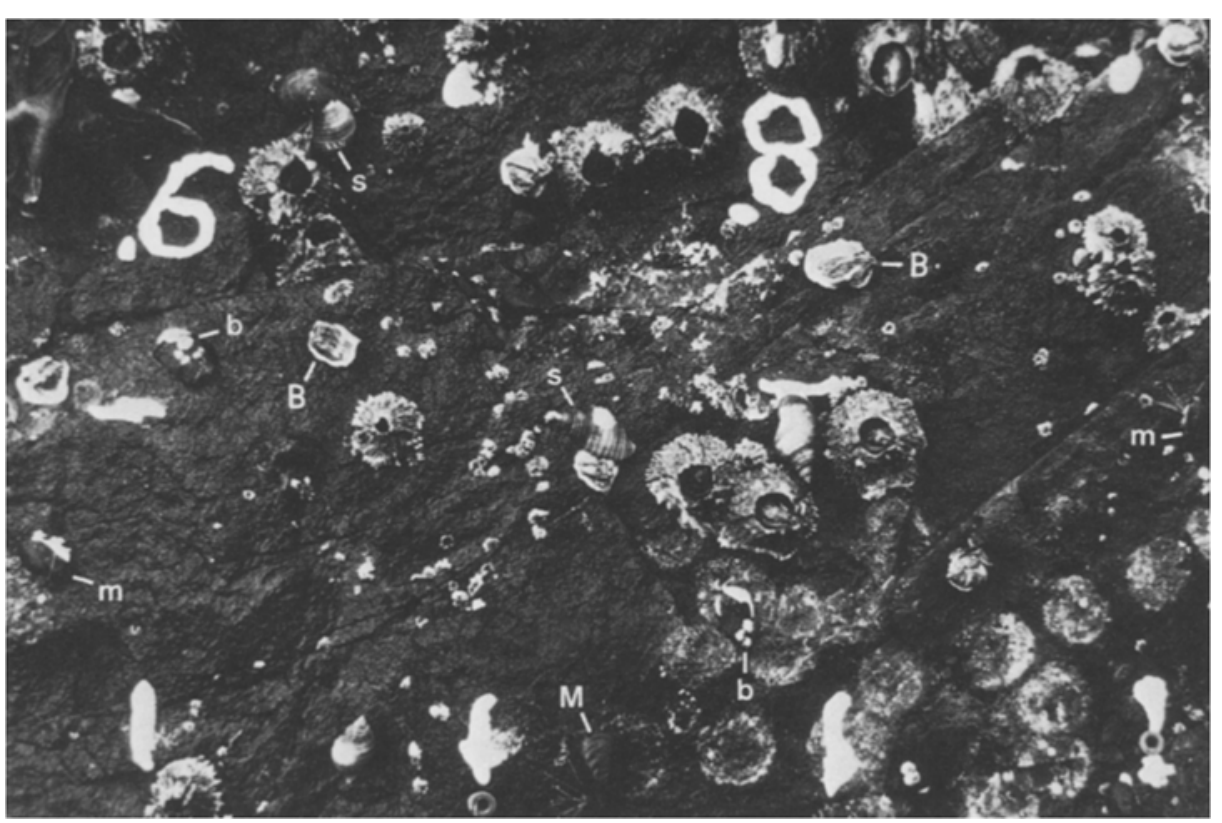

Fig. 3. Close-up of available prey and feeding snails from an arena experiment. Labelled prey include: b, small Blanus glandula; $B$, medium $B$. glandula; $m$, small Mytilus edulis; $M$, medium-small $M$. edulis; $s$, actively feeding snail
Very small barnacles attached to disarticulated $M$. edulis valves were lashed onto galvanized hardware cloth with monofilament line; the mussel valves were close enough together so snails could crawl from one to another without having to crawl across the wire mesh. Actual prey sizes and their food values are in Fig. 6 legend. 10 individually tagged $T$. emarginata within a restricted size range (range $=$ $12.5-18.4 \mathrm{~mm}$, mean $=15.8 \mathrm{~mm}$ ) were then introduced to each cage. Cages were checked periodically during the conditioning interval to ensure that an abundance of prey was always available.

At the end of the conditioning interval the cages and their contents were removed during a low tide and snails from all yield-treatments, including those in the starvation treatments (a total of 40 snails), were placed aperture down at a central point in that foraging arena. Snails were then allowed to select from four discrete prey types (listed in Fig. 6) provided within each arena in addition to naturally occurring prey, which consisted almost exclusively of low value, large $S$. cariosus $(>10 \mathrm{~mm}$ ), but also scattered small $B$. glandula and small $S$. cariosus (both approx. $2 \mathrm{~mm}$ ). The four experimentally regulated prey types were provided uniformly across the foraging arenas by glueing individual prey items to the bottom with Sea-Goin' 'Poxy Putty (Fig. 3). Items of each prey type were collected and prepared as for the cage experiments. Occasional snails leaving the foraging arenas were returned to the center of their respective arena, but those moving from one arena to the other were not disturbed, to minimize disruption of their behavior.

After releasing the snails, the arena experiments were checked twice daily, once at high tide with the aid of SCUBA and once at low tide. During these inspections, I recorded the identifying tag of each feeding Thais along with the species, size and location of the prey item being eaten. Prey apparently being attacked at high tide were verified as eaten or not eaten during the following daytime low tide. Snails still feeding at low tide were separated from their prey, whether eaten or not, and returned to the center of the foraging arenas to obtain the maximum number of feeding 'decisions' over the monitoring interval. Partially or completely consumed prey were removed from the arenas. 327 experimentally introduced prey items were provided in the July/August series over both arenas and 382 in the September/October series. The total number of prey items removed over the course of the experiment was less than $10 \%$ of those available in both series.

\section{Results}

\section{Assessment of food value}

Growth rates of all three Thais species varied as a function of predator size, prey size, prey species and tidal height; these rather extensive data are presented and discussed elsewhere (Palmer 1983). Briefly, when rank values were pooled across all predator sizes and species, the twelve prey types fell into four significantly different groups (see Fig. 13 in Palmer 1983): 1) intermediate- to large-sized (4-8 mm) $\mathrm{Ba}$ lanus glandula, which promoted the most rapid growth, 2) small- to intermediate-sized (20-43 mm) Mytilus edulis, which promoted the next most rapid rates of growth, 3) large $(>50 \mathrm{~mm}) M$. edulis and smaller $(<6 \mathrm{~mm})$ Semibalanus cariosus, which were of lower food value, and 4) larger (>6 mm) S. cariosus, which promoted the slowest growth. Although prey types of group 1 were of higher, and group 4 of lower, food value for nearly all sizes and species of Thais, the rank order of prey types within groups 2 and 3 varied rather substantially among predator species.

\section{Feeding observations at low and high tide}

The eaten and available prey were noted for a total of 45 Thais emarginata exposed while feeding at low tide at a number of localities. The mean number of available prey scored per feeding snail observed in this manner was $78 \pm 67.5$ (SD) items. These data revealed a highly significant tendency for the snails to select from available prey those of higher 'relative value' $(P<0.001$, Fig. 4). Relative value of prey was calculated as the proportion of available items with a food value higher than the expected value of 


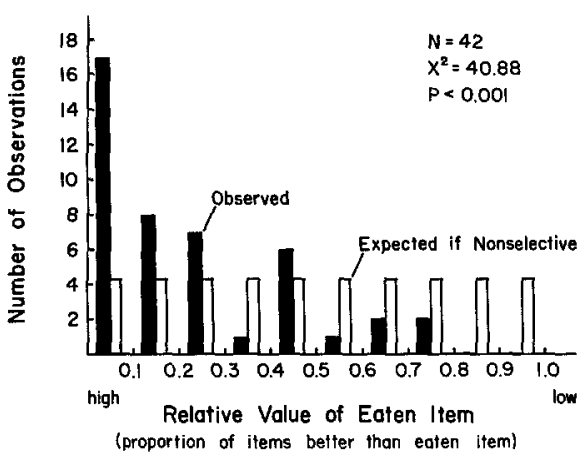

Fig. 4. Frequency distribution of feeding observations as a function of the relative value of the eaten item (defined in text). The observations were collected from a number of localities: Point George, Shaw Island $(N=3)$, Deadman Island $(N=3)$, Cattle Point, San Juan Island $(N=1)$, Waadah Island $(N=7)$, Tatoosh Island $(N=7)$, Mukkaw Bay $(N=6)$, Ocean Shores $(N=12)$, all in Washington; and Torch Bay, Alaska $(N=6)$

the eaten item. This measure of relative value was useful because it scaled out the differences in absolute values of prey among observations.

The abundance of the eaten prey type was also compared to the abundances of the available types to test for frequency-dependent foraging. Snails frequently consumed an item of the most abundant prey type available (19 of 45 cases), but in more than one third of these cases (7 of 19) the most abundant prey type was also the highest value prey type available. Hence, for these seven cases, it was not possible to distinguish between selectivity based on prey value or abundance. In the remaining 12 cases where the most abundant prey type was eaten, higher value prey were available but less abundant. In the 26 cases where a prey of the most abundant type was not eaten, the eaten type was of lower value than the most abundant type in 9 and of higher value in 17. Notably, when the eaten prey type was of lower food value (Ve) than the most abundant type ( $\mathrm{Va}$ ), the eaten prey were of only slightly lower value on average [mean $\mathrm{Va} / \mathrm{Ve}=1.1 \pm 0.11(\mathrm{SD})]$, whereas when the eaten prey type was of higher value than the most abundant type, it was of much higher value [mean $\mathrm{Ve} / \mathrm{Va}=2.5 \pm$ 1.3 (SD); ratio reversed to preserve symmetry]. This difference is highly significant $(\mathrm{T}=3.19, P=0.004)$.

Logistical constraints imposed by concurrent experiments restricted the underwater monitoring of foraging snails to one observation each for $T$. lamellosa and T. emarginata. In both observations, all encountered and rejected prey for which there were reliable estimates of food value were of lower than the item finally eaten. Although very limited, I feel these data are important because they assess the feeding behavior of unmanipulated predators in the field, where it was possible to record which prey items were rejected immediately prior to feeding. Over $2 \mathrm{~h}$ of continuous monitoring, a $36.7 \mathrm{~mm} T$. lamellosa traversed $17 \mathrm{~cm}$ and encountered and rejected 20 prey items [ 7 Balanus glandula $<2.2 \mathrm{~mm}$, (food values: -6.4 to 0.0 ), 13 Semibalanus cariosus $2.0-5.5 \mathrm{~mm}$ (food values: -6.7 to -13.1 )]; it was still searching when observation ceased. The same snail was located the following morning feeding on a $3.0 \mathrm{~mm} \mathrm{~B}$. glandula (food value: 9.9). Although there was no record of other prey which may have been encountered in the interim, the eaten barnacle was of higher value than any of the items encountered while the snail was being monitored.
Table 1. Proportions of four prey types eaten by Thais lamellosa (mean shell length $=36 \mathrm{~mm}$, range $=31.1-40.3 \mathrm{~mm}$ ) after having been conditioned at various yields. The data have been pooled from both replicates at each tidal height and from both tidal heights at which the experiments were conducted $(0.0 \mathrm{ft}, 0.0 \mathrm{~m}$ and $+2.0 \mathrm{ft}$, $+0.6 \mathrm{~m}) . \mathrm{Bg}=$ Balanus glandula. $\mathrm{Sc}=$ Semibalanus cariosus, $\mathrm{Me}=$ Mytilus edulis, $\mathrm{Sm}=$ small, $\mathrm{Med}=$ medium. $\mathrm{M}-\mathrm{S}=$ medium-small, $\mathrm{Lg}=$ large. Barnacle sizes are opercular diameters, mussel sizes are shell lengths. Actual prey sizes indicated in parentheses except: $\mathrm{M}-\mathrm{S} M$. edulis $=25 \mathrm{~mm}$. Expected yields represent the mean percent change in body weight over 30 days and were obtained from (Palmer 1983). Tabled yield estimates are the average of the yields from both tidal heights. Proportions correspond to the proportions of a given prey type eaten from both tidal heights out of all prey items eaten by snails from a given conditioning treatment. $\mathrm{N}=$ total number of prey eaten or attacked by snails from that conditioning treatment. $X^{2}=$ Chi square statistic obtained from a contingency table analysis. $\mathrm{P}=$ probability

\begin{tabular}{|c|c|c|c|c|c|c|}
\hline \multirow{2}{*}{$\begin{array}{l}\text { Conditioning } \\
\text { prey type } \\
\text { and size } \\
(\mathrm{mm})\end{array}$} & \multirow{2}{*}{$\begin{array}{l}\text { Ex- } \\
\text { pec- } \\
\text { ted } \\
\text { yield }\end{array}$} & \multicolumn{5}{|c|}{$\begin{array}{l}\text { Proportions of prey } \\
\text { selected in observation cages }\end{array}$} \\
\hline & & $\begin{array}{l}\text { Med } \\
\mathrm{Bg}\end{array}$ & $\mathrm{Sm} \mathrm{Me}$ & $\begin{array}{l}\mathrm{M}-\mathrm{S} \\
\mathrm{Me}\end{array}$ & $\mathrm{Sm} \mathrm{Bg}$ & $N$ \\
\hline None (starved) & - & 0.70 & 0.04 & 0.02 & 0.24 & 134 \\
\hline $\mathrm{Sm} \mathrm{Bg}(1.7)$ & -1.7 & 0.77 & 0.01 & 0.01 & 0.21 & 90 \\
\hline $\mathrm{Sm} \mathrm{Sc}(4.0)$ & $8.1^{\mathrm{a}}$ & 0.67 & 0.03 & 0.02 & 0.29 & $66^{\mathrm{a}}$ \\
\hline $\mathrm{Sm} \mathrm{Me} \mathrm{(15)}$ & 14.7 & 0.50 & 0.10 & 0.20 & 0.20 & 80 \\
\hline Med Bg (5.0) & 24.7 & 0.76 & 0.09 & 0.09 & 0.07 & 46 \\
\hline \multicolumn{7}{|l|}{ Pooled } \\
\hline \multirow{3}{*}{$\begin{array}{l}\text { All non-mussel } \\
\text { Mussel only }\end{array}$} & & 0.72 & 0.04 & 0.03 & 0.22 & 336 \\
\hline & & 0.50 & 0.10 & 0.20 & 0.20 & 80 \\
\hline & & \multicolumn{5}{|c|}{$X^{2}=42.5^{b} \quad P<0.001$} \\
\hline
\end{tabular}

a Data from the low tidal height only $(0.0 \mathrm{ft}, 0.0 \mathrm{~m})$

b From pooled data only

A $12.0 \mathrm{~mm} T$. emarginata encountered and rejected 12 items [2 Chthamalus dalli $2.0 \mathrm{~mm}$ (extrapolated food value: approx. 104), $6 S$. cariosus $2.7-7.0 \mathrm{~mm}$ (food values: 5.4 to 17.4) and 4 Mytilus edulis $2.0-4.0 \mathrm{~mm}$ (extrapolated food value: approx. 100)] before it attacked a $1.6 \mathrm{~mm} \mathrm{B.} \mathrm{glandula}$ (food value: 95.5 ). The following low tide, the allegedly attacked barnacle was verified as consumed and the snail was relocated consuming a $7.0 \mathrm{~mm} \mathrm{M}$. edulis (extrapolated food value: approx. 100). Both these eaten items were of comparable values, but because both the rejected and consumed mussels were 3 to 10 times smaller than the smallest size class available in the controlled feeding experiments ( $20 \mathrm{~mm}$; Palmer 1983), the estimates of their expected food value were very approximate. The expected values of the two Chthamalus encountered and rejected were also approximate since they estimated from snails grown at a different tidal height than that from which the values of the other prey were determined.

\section{Experiments with manipulated yields}

In the cage experiments, differences between replicate cages in the frequencies of eaten prey, both at the same and at different tidal heights, were significant in only one of 23 pairs $(P=0.013 ; P>0.1$ for all others), so all replicates for each snail species were pooled (Tables 1-3). Differences in prey selection among the yield-treatment groups, including the starvation treatment, were slight; all three predator spe- 
Table 2. Proportions of four prey types eaten by Thais canaliculata (mean shell length $=24 \mathrm{~mm}$, range $=22.0-27.2 \mathrm{~mm}$ ) after having been conditioned at various yields. The data were pooled as for T. lamellosa. See Table 1 legend for abbreviations and details

\begin{tabular}{|c|c|c|c|c|c|c|}
\hline \multirow{2}{*}{$\begin{array}{l}\text { Conditioning } \\
\text { prey type } \\
\text { and size } \\
(\mathrm{mm})\end{array}$} & \multirow{2}{*}{$\begin{array}{l}\text { Ex- } \\
\text { pec- } \\
\text { ted } \\
\text { yield }\end{array}$} & \multicolumn{5}{|c|}{$\begin{array}{l}\text { Proportions of prey } \\
\text { selected in observation cages }\end{array}$} \\
\hline & & $\begin{array}{l}\text { Med } \\
\mathrm{Bg}\end{array}$ & $\begin{array}{l}\mathrm{M}-\mathrm{S} \\
\mathrm{Me}\end{array}$ & $\mathrm{Sm} \mathrm{Me}$ & $\mathrm{Sm} \mathrm{Bg}$ & $N$ \\
\hline None (starved) & - & 0.55 & 0.06 & 0.15 & 0.24 & 96 \\
\hline $\mathrm{Sm} \mathrm{Bg}(1.7)$ & 18.3 & 0.47 & 0.11 & 0.27 & 0.26 & 45 \\
\hline $\operatorname{Sm~Sc}(4.0)$ & $72.1^{\mathrm{a}}$ & 0.46 & 0.12 & 0.15 & 0.27 & $26^{\mathrm{a}}$ \\
\hline $\mathrm{M}-\mathrm{S} \mathrm{Me}(25)$ & 73.7 & 0.44 & 0.20 & 0.20 & 0.17 & 41 \\
\hline Med Bg (5.0) & 107.4 & 0.83 & 0.08 & 0.00 & 0.18 & 24 \\
\hline \multicolumn{7}{|l|}{ Pooled } \\
\hline \multirow{3}{*}{$\begin{array}{l}\text { All non-mussel } \\
\text { Mussel only }\end{array}$} & & 0.55 & 0.08 & 0.16 & 0.20 & 191 \\
\hline & & 0.44 & 0.20 & 0.20 & 0.17 & 41 \\
\hline & & \multicolumn{5}{|c|}{$X^{2}=5.38^{\mathrm{b}}$} \\
\hline
\end{tabular}

a Data from the low tidal height only $(0.0 \mathrm{ft}, 0.0 \mathrm{~m})$

b From pooled data only

Table 3. Proportions of four prey types eaten by Thais emarginata (mean shell length $=14 \mathrm{~mm}$, range $=12.3-15.5 \mathrm{~mm}$ ) after having been conditioned at various yields. The data were pooled from both replicates at the high tidal height $(+4.0 \mathrm{ft},+1.3 \mathrm{~m})$. See Table 1 legend for abbreviations and details

\begin{tabular}{|c|c|c|c|c|c|c|}
\hline \multirow{2}{*}{$\begin{array}{l}\text { Conditioning } \\
\text { prey type } \\
\text { and size } \\
(\mathrm{mm})\end{array}$} & \multirow{2}{*}{$\begin{array}{l}\text { Ex- } \\
\text { pec- } \\
\text { ted } \\
\text { yield }\end{array}$} & \multicolumn{5}{|c|}{$\begin{array}{l}\text { Proportions of prey } \\
\text { selected in observation cages }\end{array}$} \\
\hline & & $\begin{array}{l}\text { Med } \\
\text { Bg }\end{array}$ & $\mathrm{Lg} \mathrm{Bg}$ & $\mathrm{Sm} \mathrm{Me}$ & $\mathrm{Sm} \mathrm{Bg}$ & $N$ \\
\hline None (starved) & - & 0.37 & 0.07 & 0.27 & 0.30 & 30 \\
\hline $\mathrm{Sm} \mathrm{Bg}(1.7)$ & 49.8 & 0.53 & 0.13 & 0.13 & 0.20 & 15 \\
\hline Sm Me (15) & 63.2 & 0.54 & 0.00 & 0.31 & 0.15 & 13 \\
\hline $\operatorname{Lg} B g(8.0)$ & 82.8 & 0.55 & 0.00 & 0.18 & 0.27 & 11 \\
\hline Med Bg $(5.0)$ & 101.9 & 0.67 & 0.00 & 0.00 & 0.33 & 3 \\
\hline \multicolumn{7}{|l|}{ Pooled } \\
\hline \multirow{3}{*}{$\begin{array}{l}\text { All non-mussel } \\
\text { Mussel only }\end{array}$} & & 0.46 & 0.07 & 0.20 & 0.27 & 59 \\
\hline & & 0.54 & 0.00 & 0.31 & 0.15 & 13 \\
\hline & & \multicolumn{5}{|c|}{$X^{2}=2.14^{\mathrm{a}} \quad P=0.46$} \\
\hline
\end{tabular}

a From pooled data only

cies from all yieid-treatments preferentially consumed medium-sized $B$. glandula, the most valuable prey available. However, some of the snails conditioned on small $M$. edulis behaved differently. The frequencies of pooled observations for $T$. lamellosa from the non-mussel treatments were significantly different from those conditioned on mussels $\left(X^{2}=\right.$ 42.5, $P<0.001$, Table 1). T. canaliculata conditioned on $M$. edulis exhibited a similar, but statistically insignificant bias toward consuming mussels $\left(X^{2}=5.38, P=0.15\right.$, Table 2). $T$. emarginata conditioned on $M$. edulis, however, exhibited no greater tendency to choose mussels than did snails from the other treatments $\left(X^{2}=2.14, P=0.46\right.$, Table 3$)$.

In the arena experiments, differences between the two replicate series were not significant so the results were pooled. Snails from both low-yield treatments consumed experimentally introduced prey in proportions almost ident- ical to those consumed by snails from the starvation treatment (solid bars, Figs. 6a-6c). Snails conditioned at a high yield, however, consumed almost exclusively the highest value prey type available (solid bars, Fig. 6 d). Thus only exposure to a high yield (high potential rate of growth) altered feeding behavior when compared to starved animals.

\section{Discussion}

A key limitation of most foraging studies is that in multiprey systems the food value of prey (e.g. ability to promote growth or influence reproductive output) is rarely measured directly (Schluter 1981). I addressed this problem by growing the consumers on pure diets of various prey types supplied ad libitum (Palmer 1983). Growth rates were related to age at maturation and reproductive output, thus food value could be related directly to fitness (Palmer 1983), a necessary requirement of optimization models (Lewontin 1979). Further, using these growth rates, prey could be ranked in units directly relevant to the predator; no assumptions about the conversion of prey calories to calories usable by the predator were necessary. The only assumption required was that predators could distinguish among prey types based upon their ability to promote growth. Using these measures of food value, both natural and experientally obtained feeding observations can be compared quantitatively to three expected patterns of prey selection: nonselective (Griffiths 1975), frequency-dependent (Fullick and Greenwood 1979; Greenwood and Elton 1979; Murdoch 1969) and energy maximization (optimal: Charnov 1976; Pulliam 1974; Schoener 1971).

\section{Reliability of food value estimates}

Overall rates of feeding in the yield-manipulation experiments suggest that growth rates were a rather remarkable predictor of food value as preceived by the snails. In all four series (all three Thais species in the cage experiments and $T$. emarginata in the arena experiments), snails conditioned at a lower average yield consumed prey at a significantly higher rate during the subsequent monitoring interval than those conditioned at a higher yield (Fig. 5). Presumably, overall feeding rate is a reflection of the 'hunger' state of a consumer. Since some physiological manifestation of 'hunger' (e.g. blood sugar) is probably the mechanism by which consumers assess their average rate of energy intake or yield (Dethier 1976), those experiencing a lower average yield should be 'hungrier'. Thus, the consistently significant, negative relationships between the food value of the prey type used to condition snails at different yields and their subsequent feeding rate (Fig. 5) indicate that both food value and average yield were predicted accurately from the experimentally determined rates of snail growth (Palmer 1983).

\section{Feeding observations at low and high tide}

The feeding observations at low tide provided fairly strong evidence that $T$. emarginata were capable of recognizing the expected values and choosing the higher value prey (Fig. 4). In addition, although the prey item consumed was often of the most common type (size class of a particular species), in the majority of the cases (26 of 45 ) it was not, indicating that prey selection was not purely frequencydependent. Interpretation is difficult for the 12 cases where 


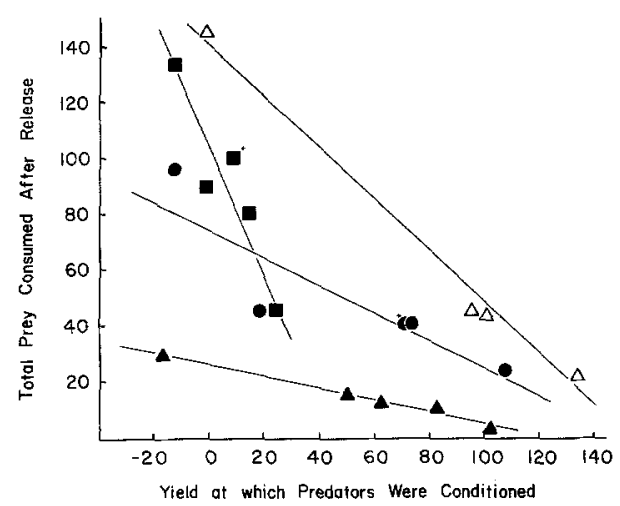

Fig. 5. Total number of prey items consumed by Thais following release from the conditioning cages as a function of the average yield at which the snails were conditioned. The data are from Tables 1-3 and Fig. 6. Solid symbols represent data from the cage experiments: squares $=$ Thais lamellosa $\left(\mathrm{r}^{2}=0.855 ; P=0.025 ; 20\right.$ snails per point over 12 days $)$, circles $=T$. canaliculata $\left(r^{2}=0.774\right.$ $P=0.049 ; 20$ snails per point over 12 days), triangles $=T$. emarginata $\left(\mathrm{r}^{2}=0.978 ; P=0.001 ; 10\right.$ snails per point over 12 days $)$. Open symbols are for $T$. emarginata from the arena experiments $\left(\mathrm{r}^{2}=\right.$ $0.989 ; P=0.011 ; 20$ snails per point over 13 days). ' + ' $=$ Pooled consumption rate estimated from the consumption rate at the low tidal level plus the expected proportion consumed at the mid level as determined from the controlled growth experiments (Palmer 1980). Lines fitted by eye. Note that the slope differences among the different groups are not meaningful because the absolute values for yield are not directly comparable among predator species; yield was measured as percent body weight gain over a fixed time interval and percent weight gain varies depending on the starting sizes of the snails - snails from different groups were of different initial sizes

higher value prey types were available but less abundant than the prey type eaten; they suggest frequency-dependent foraging but cannot rule out prey selection based upon energetic considerations because the acceptability threshold, if the predator was foraging optimally, was unknown. Similarly, the 9 cases where the prey item being eaten was of lower value than the most abundant type suggest that the predators were not foraging optimally, but again, without knowing the acceptability thresholds no conclusion may be drawn. Overall, these results are in closest qualitative agreement with the expectations of the optimal diet model; they do not, however, provide a convincing test.

Such feeding observations on unmanipulated consumers have the advantage of avoiding potential artifacts introduced by experimental manipulations, but, as apparent above, they usually lack the precision required for quantitative discrimination among hypotheses. However, one powerful observation test of the optimal diet model is to monitor directly a predator foraging in the field and record all prey items encountered and rejected before feeding is initiated. The model predicts that during a given foraging bout, prey types of a particular value should either always be eaten when encountered or should never be eaten (Charnov 1976, Pulliam 1974, Schoener 1971). Because of its threshold nature, a specific prediction is that the eaten item should be the most valuable one encountered since the last eaten item or since searching was initiated. This test may be performed even if the acceptability threshold is unknown. It assumes that: 1) re-evaluation of average yield is slow relative to encounter rates, 2) the expected value of prey types has been assessed accurately and remains con- stant over the duration of the foraging bout, 3 ) it is possible to score rejections reliably, and 4) that there is little or no cost to rejection.

The observations of individual snails foraging at high tide suggest that Thais will reject lower value prey items in anticipation of locating one of higher expected value, a behavior not consistent with non-selective or frequencydependent models of prey selection. The generality of this conclusion is rather uncertain because it is based on observations of only two snails, but I present this type of data because it demonstrates the potential for a powerful observational test of the threshold nature of the optimal foraging model. More observations of this type could provide considerable insight into the factors influencing prey selection in the field.

\section{Experiments with manipulated yields}

Both the cage and arena experiments were designed to assess the impact of immediate past feeding history on subsequent prey selection. By controlling experimentally the range of feeding regimes experienced, it was possible to separate the effects on prey choice of 1) starvation, 2) exposure to a high abundance of specific prey types, and 3) exposure to different levels of average yield in terms of potential growth or reproductive output (Palmer 1983). An important assumption of these experiments was that snails did not reevaluate their estimate of average yield during the monitoring interval following release. Data from other experiments suggest that Thais require more than 14 days before significant change in this estimate occurs (Palmer 1980); reevaluation may take up to $4 \mathrm{wks}$ in the Australian thaidid Lepsiella vinosa (Bayliss 1982, p. 221) and in the California thaidid Acanthina spirata (D.M. Perry, unpublished).

The starvation treatments in both the cage and arena experiments were important because they provided the null frequencies (frequencies of prey eaten if non-selective) against which the frequencies of prey eaten following particular feeding regimes could be compared. I have assumed here that the approximately 30 days starvation these snails experienced was sufficient to reduce substantially or to eliminate any effects of their feeding history prior to collection for the experients. Further I have assumed that the proportions of the prey selected by animals from the starved treatments in both experiments provided a reasonable estimate of the proportions in which prey were actually encountered by the snails. Such an estimate is critical since the availabilities of prey perceived by the predator may differ significantly from prey abundances as measured by the investigator (B. Menge 1972; J. Lubchenco Menge 1974; Milinski and Heller 1978; Murdoch et al. 1975; Newsome and Gee 1978; Royama 1970).

The cage experiments were designed to test a quantitative prediction of optimal diet models: snails having experienced experimentally regulated, high average yields should feed exclusively on high value prey. However, two unanticipated artifacts substantially reduced their discriminatory power. First, the spacing of individual prey on the $4 \times 5 \mathrm{~cm}$ grid appears to have placed more than one item at a time within the snail's chemosensory perceptive field, allowing them to recognize the presence of higher value prey a short distance away. Second, this artifact was aggravated by a weak but sustained long-shore current $(1-2 \mathrm{~cm} / \mathrm{s}$, Palmer 


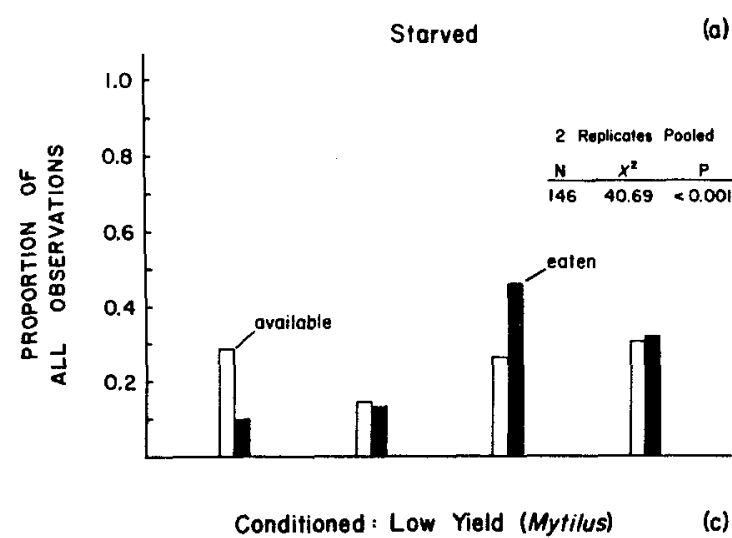

(a)
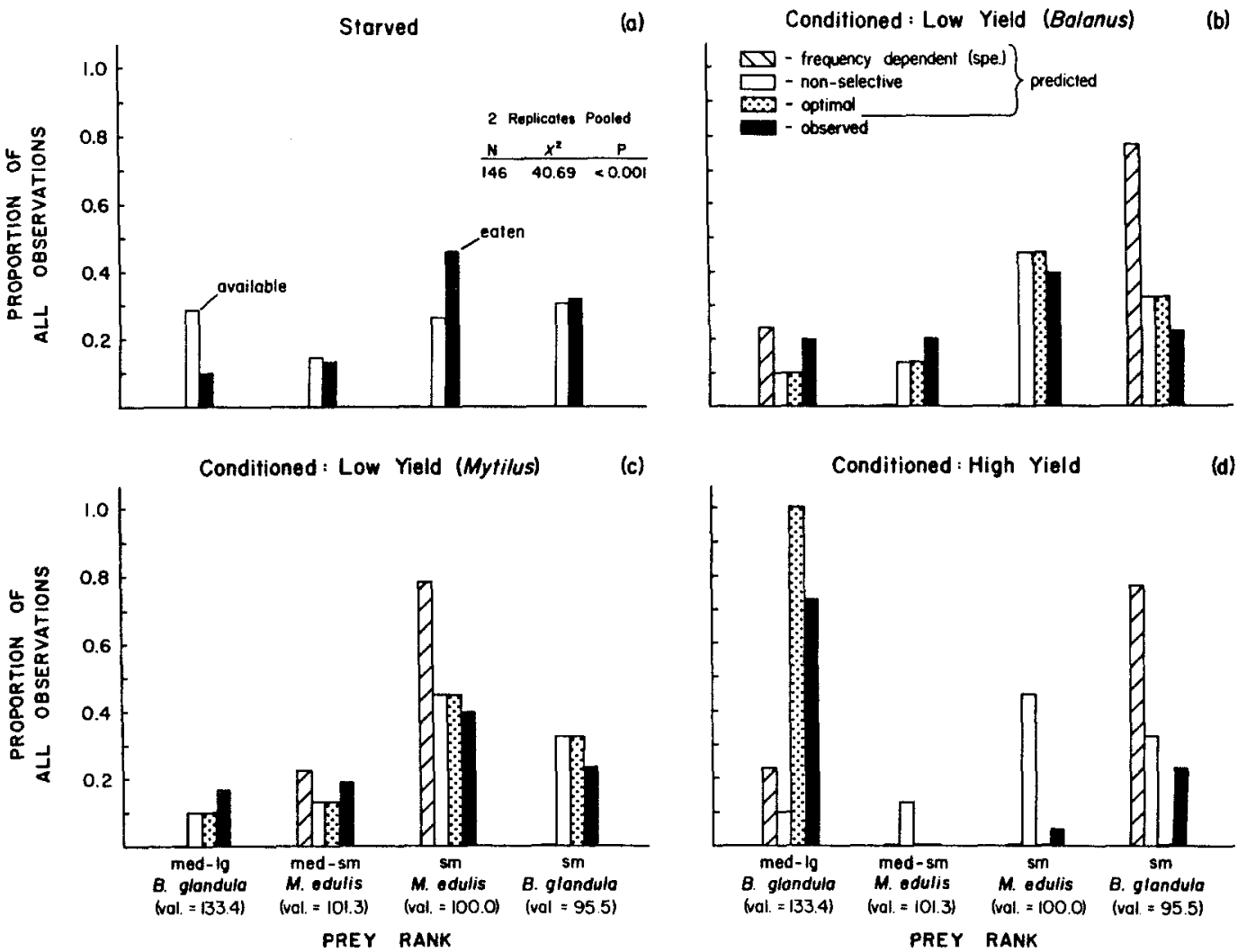

Fig. 6a-d. Predictions and results of the arena experiments with Thais emarginata. Snails conditioned at the low yield on Balanus glandula were fed small barnacles (approx. $2 \mathrm{~mm}$, growth equivalent $=95.5$; Palmer 1983). Those conditioned at the low yield on Mytilus edulis were fed medium-small mussels (approx. $30 \mathrm{~mm}$ growth equivalent $=101.3$ ). Snails conditioned at the high yield were fed intermediate sized B. glandula $(46 \mathrm{~mm}$, growth equivalent $=133.4)$. a Comparison of proportions eaten (solid bars) versus proportions of each prey type available (open bars) in the foraging arenas for snails that were starved over the conditioning interval. b-d Comparisons of proprotions of prey eaten versus predicted proportions for each of three alternative foraging hypotheses [frequency-dependent foraging on prey species (diagonally marked bars), non-selective foraging (open bars), and optimal foraging (stippled bars)]. The total number of observations for each treatment were: a number available $=709$, number observed $=146, \mathbf{b}$ number observed $=36, \mathbf{c}$ number observed $=$ 43 , $\mathbf{d}$ number observed $=22$

1980) where these cages were located, which probably also allowed prey to be detected from a greater distance (e.g., see Kohn 1961, Snyder and Synder 1971). As a consequence nearly all snails, including starved animals and regardless of prior conditioning, fed predominantly on the highest value prey (intermediate sized $B$. glandula, Tables $1-3$ ). Such a response is consistent with the optimal diet model, however, the influence of average yield on prey choice could not be evaluated quantitatively.

In spite of the apparent artifacts, some qualitative conclusions may still be drawn from the cage experiments. Both $T$. lamellosa and $T$. canaliculata conditioned at a high yield on medium-sized $B$. glandula consumed fewer low value, small B. glandula than snails conditioned at any of the lower yields (Tables 1 and 2). This decreased consumption of lower value prey types is not consistent with the non-selective foraging hypothesis, since past feeding history should not have influenced the pattern of prey selection. The frequency-dependent foraging model was also not supported, as no treatment group other than those conditioned on the high-value, medium-sized $B$. glandula specialized on prey to which they had been conditioned. The apparent 'specialization' by the animals conditioned on mediumsized $B$. glandula is not evidence of frequency-dependent selection because snails from all treatment groups, whether starved or conditioned on mussels or barnacles, consumed this prey most frequently (Tables 1-3). However, in nearly all treatment groups for all three Thais species, some of each of the available prey types were consumed. Snails conditioned at the highest yield were least likely to consume low-value prey, but the optimal diet models predicts that none should have been consumed. Thus, although the direction of the change in diet as a function of immediate past feeding history was predicted by models maximizing the rate of energy intake, a result inconsistent with alternative models, the results did not agree in detail with the quantitative predictions.

The results of the arena experiments, which were free of most of the artifacts of the cage experiments, were much clearer. First, there was a highly significant difference between the proportions of prey types eaten by starved animals and the proportions of each experimental prey type actually present (i.e., glued into the intertidal; $P<0.001$, Fig. 6a). Assuming non-selective feeding by starved snails, this suggests that the chemosensory 'reaction distance' (Charnov 1976) was different for different prey types (see also Blake 1960); prey detected from a greater distance were more likely to have been encountered. The rather low frequency with which high-value $B$. glandula were eaten further suggests that available prey were encountered one at a time, in contrast to the difficulties encountered with the cage experiments. Since starved Thais appeared to per- 
ceive prey in proportions other than the proportions provided experimentally in the areas, the proportions of prey consumed in all the subsequent treatments were compared to these perceived proportions.

The hypothesis of non-selective foraging (open bars in Figs. $6 \mathrm{~b}$ to $6 \mathrm{~d}$ ) is inadequate to account for the results observed in the arena experiments. Although the proportions of prey eaten agreed closely with those expected by non-selective foraging in treatments $b$ and $c$ (Fig. 6), the same result was predicted by the optimal diet model because there was so little difference between the average yields of these low-yield treatments. In treatment $\mathrm{d}$, however, the proportions eaten departed markedly from those expected if feeding was non-selective; approximately $70 \%$ of the snails were feeding on intermediate sized Balanus glandula, the highest value prey.

The hypothesis of frequency-dependent foraging on prey types (particular size of a particular species) may be ruled out for this system. An extreme version of this hypothesis predicts that the predator should specialize on what it perceives to be the most abundant prey type. Only in treatment $d$ was there evidence of preferential feeding on the conditioning prey type, but this would also have been expected from the optimal foraging hypothesis. In treatments $b$ and $c$ there was no evidence for preferential feeding by prey type; all available prey types were eaten in approximately the proportions they were encountered.

The hypothesis of frequency-dependent foraging on prey species (diagonally marked bars, Fig. 6) may also be ruled out. The strongest evidence against this hypothesis derives from a comparison of treatments $b$ and $d$ (Fig. 6). If preferential feeding on prey species occurred, then snails in both treatment groups should have selected prey in similar proportions since both had been conditioned on Balanus glandula; this was clearly not the case (compare solid bars in $b$ and $d$ ). Relaxing the rather extreme expectation of complete specialization of these two hypotheses, there is still no compelling evidence for preferential feeding on either the prey type or species on which the predator had been conditioned in treatments $b$ and $c$.

The results of from all the treatment groups were most consistent with the predictions of the optimal foraging hypothesis (stippled bars, Fig. 6). Since food was supplied in the conditioning cages such that snails could feed ad libitum, search time would have been minimal. Consequently, the average yield (energy or growth potential obtained per unit foraging time) experienced by the snails in the conditioning cages should have been close to, although probably less than, the expected value energy or growth potential obtained per unit handling time) of the prey type on which the snails were conditioned. If the animals were foraging optimally, they should have rejected all prey types whose value was less than the average yield they had been experiencing. In treatments $b$ and c, snails consumed prey in the proportions in which they were encountered, as predicted. In treatment $d$, though not feeding exclusively on this prey type, the snails were feeding on the highest value prey at a much higher frequency than the other prey types. Further, the dramatic departure of the pattern of prey selection in treatment $d$ from the patterns in treatments a-c suggests strongly that the snails in this treatment were actively rejecting prey of lower value.

Although the optimal diet model was the most successful of those examined in predicting the results of the arena
Table 4. Mean squared deviations of observed from expected frequencies for each of four alternative foraging hypotheses, following three yield treatments (treatments b-d of Fig. 6). The total observations for each treatment have been scaled to the same value prior to computing the mean squared deviations (that of the smallest sample, $N=22$ in treatment d) to avoid the sample-size dependent bias to which this descriptive statistic is sensitive

\begin{tabular}{lllll}
\hline Hypothesis & \multicolumn{2}{l}{ Treatment } & \multicolumn{2}{l}{$\begin{array}{l}\text { Aver- } \\
\text { age }\end{array}$} \\
\cline { 2 - 5 } & $\begin{array}{l}\text { Low } \\
\text { yield } \\
\text { (Balanus) }\end{array}$ & $\begin{array}{l}\text { Low } \\
\text { yield } \\
\text { (Mytilus) }\end{array}$ & $\begin{array}{l}\text { High } \\
\text { yield } \\
\text { (Balanus) }\end{array}$ & \\
\hline $\begin{array}{l}\text { Frequency-dependent } \\
\text { (type) }\end{array}$ & 107.75 & 109.81 & 15.52 & 77.69 \\
$\begin{array}{l}\text { Frequency-dependent } \\
\text { (spe) }\end{array}$ & 66.01 & 28.64 & 65.84 & 53.50 \\
$\begin{array}{l}\text { Non-selective } \\
\text { Optimal foraging }\end{array}$ & 6.35 & 1.92 & 70.41 & 26.13 \\
\hline
\end{tabular}

experiments, the statistical significance of these results is uncertain since for all but the non-selective hypothesis, there were one or more expected frequencies of zero. In such cases, any observation, strictly speaking, invalidates the attendant hypothesis. Thus, I had to resort to a measure of relative agreement, mean squared deviation (MSD), to compare the observed results with the predictions, admitting $a$ priori that the predictions of the various hypotheses were approximate. For all hypotheses except optimal foraging (Table 4), the observed values from one or more treatment groups differed from the predicted values by an amount greater than 15 times that of the MSD between the observed values from the two replicate series ( 4.44 between the 16 pairs). No treatment group differed from the frequencies predicted by optimal foraging by more than a factor of four.

\section{Learning and risk}

Although energetic considerations clearly influenced prey choice by Thais, two additional factors implicated by this study include learning, and risk of predation or physiological stress. Evidence for learning derives from the cage experiments, in which both $T$. lamellosa, and to a much lesser extent T. canaliculata, appeared to exhibit some degree of 'ingestive conditioning' (Wood 1968). This phenomenon, which has been observed in several gastropod species (Bayliss 1982; Hall et al. 1982; Wood 1968), is not consistent with the expectations of the optimal diet model because expected food values are assumed to remain constant within a foraging session. In particular, the optimal diet model cannot account for why starved, presumably non-selective snails, did not consume $M$. edulis more frequently than snails conditioned on mussels.

Of interest, the degree of 'ingestive conditioning' was correlated with the magnitude of the difference in overall expected values among these prey, which was also correlated with past experience. In other words, T. lamellosa, the species exhibiting the strongest conditioning when exposed to $M$. edulis, grew more slowly on $M$. edulis relative to $B$. glandula than either $T$. canaliculata or $T$. emarginata (Palmer 1983). It also rarely consumed $M$. edulis in the field. Thus, $T$. lamellosa may not have recognized the value of an unfamiliar low-quality prey, and 'learned' to do so 
only with experience. Alternatively, mussel-conditioned snails in the cage experiments may have consumed mussels at a higher rate because of increased handling efficiency gained from past experience, as has been demonstrated for the moon snail, Polinices duplicatus, feeding on Mya arenaria (Edwards and Huebner 1977). Such learning may be an important element affecting the ranking and hence subsequent choice of prey (Hughes 1979; Jaeger and Rubin 1982).

Evidence that some perception of risk may have influenced prey choice comes from the arena experiments, where small $B$. glandula were consumed in higher frequencies than predicted by the optimal diet model (Fig. 6d). This may indicate that Thais are less capable of distinguishing among different sizes of prey than among different prey species. Alternatively, the desirability of small $B$. glandula may have varied due to changes in anticipated risk over a tidal cycle because of their short handling time. Since handling times are longer for larger barnacles (Carefoot 1977; Emlen 1966; Palmer 1980), a snail attempting to consume one during a falling tide may be subject to the risks attendant with exposure at low tide (temperature and desiccation stress, Bertness and Schneider 1976; vulnerability to avian predators, Zach 1978). Consumption of small barnacles which have shorter handling times may not entail such risks, as demonstrated rather dramatically by J. Lubchenco Menge (1974). This interpretation implies that the perceived risks in the field were greater than in the growth cages from which prey values were determined, since, if incorporating risk, the snails in the growth cages should have spent less time in a feeding position. Including risk in foraging models (Emlen 1966, 1973; J. Lubchenco Menge 1974; Milinski and Heller 1978; Sih 1980, 1982) may increase their utility in predicting prey selection in the field.

Acknowledgements. This work formed part of a dissertation submitted in partial fulfillment of the requirements for a $\mathrm{PhD}$ at the University of Washington. As with most dissertation studies, I am indebted to many people in many ways as I have acknowledged elsewhere (Palmer 1980). Thanks are due to my reading committee, R.T. Paine ('el Pavo Grandé'), T.W. Schoener and R. R. Strathmann, for their efforts to keep the dissertation literate and logically consistent. Additional suggestions on the MS from K. Irons, R.B. Lowell, R. Ostfeld, L, Richards, C.M. Young and several reviewers were also helpful. I am particularly grateful to R.T. Paine for his sustained, enthusiastic support and persistent, thoughtful harassment throughout the duration of the study. NSF grants OCE74-02307 and OCE-77-26901-02 to R.T. Paine provided logistical and salary support without which a study of this magnitude could not have been completed. Preparation and publication expenses were defrayed by NSERC operating grant A7245 to the author.

\section{References}

Abbott RT (1974) American Seashells. Van Nostr Reinhold Co 663 $\mathrm{pp}$

Bayliss DE (1982) Switching by Lepsiella vinosa (Gastropoda) in South Australian mangroves. Oecologia (Berlin) 54:212-226

Belovsky GE (1978) Diet optimization in a generalist herbivore: the moose. Theor Pop Biol 14:105-134

Bertness MD, Schneider DE (1976) Temperature relations of Puget Sound thaids in reference to their intertidal distribution. Veliger $19: 47-58$

Blake JW (1960) Oxygen consumption of bivalve prey and their attractiveness to the gastropod Urosalpinx cinerea. Limnol Oceanogr 5:273-280
Carefoot TH (1977) Pacific Seashores. Univ of Washington Press, Seattle, $208 \mathrm{pp}$

Charnov EL (1976) Optimal foraging: attack strategy of a mantid Amer Natur 110:141-151

Dethier VG (1976) The Hungry Fly. Harvard University Press, Cambridge

Draulans D (1982) Foraging and size selection of mussels by the tufted duck, Aythya fuligula. J Anim Ecol 51:943-956

Ebersole JP, Wilson JC (1980) Optimal foraging: the responses of Peromyscus leucopus to experimental changes in processing time and hunger. Oecologia (Berlin) 46:80-85

Edwards DC, Huebner JD (1977) Feeding and growth rates of Polinices duplicatus preying on Mya arenaria at Barnstable Harbor, Massachusetts. Ecology 58:1218-1236

Elner RW, Hughes RN (1978) Energy maximization in the diet of the shore crab Carcinus maenas. J Anim Ecol 47:102-116

Emlen JM (1966) Time energy and risk in two species of carnivorous gastropods. PhD Dissertation, Univ Wash, Seattle $128 \mathrm{pp}$

Emlen JM (1973) Ecology: An Evolutionary Approach, AddisonWesley, Reading, $493 \mathrm{pp}$

Estabrook GF, Dunham AE (1976) Optimal diet as a function of absolute abundance, relative abundance, and relative value of available prey. Amer Natur 110:401-413

Fullick TG, Greenwood JJD (1979) Frequency dependent food selection in relation to two models. Amer Natur 113:762-765

Goss-Custard JD (1977) Optimal foraging and size selection of worms by redshank, Tringa totanus, in the field. Anim Behav $25: 10-29$

Greenwood JJD, Elton RA (1979) Analysing experiments on frequency-dependent selection by predators. J Anim Ecol 48:721-738

Griffiths D (1975) Prey availability and the food of predators. Ecology 56:1209-1214

Hall SJ, Todd CD, Gordon AD (1982) The influence of ingestive conditioning on the prey species selection in Aeolidia papillosa (Mollusca Nudibranchia). J Anim Ecol 51:907-921

Hughes RN (1979) Optimal diets under the energy maximization premise: the effects of recognition time and learning. Amer Natur 113:209-221

Hughes RN (1980) Optimal foraging theory in the marine context. Oceanogr Mar Biol Ann Rev 18:423-481

Jaeger RG, Rubin AM (1982) Foraging tactics of a terrestrial salamander: judging prey profitability. J Anim Ecol 51:167-176

Kohn AJ (1961) Chemoreception in gastropod molluscs. Amer Zool 1:291-308

Krebs JR, Erickson T, Webber MT, Charnov EL (1977) Optimal prey selection in the great tit (Parus major). Anim Behav $25: 30-38$

Lacher TE Jr, Willig MR, Mares MA (1982) Food preference as a function of resource abundance with multiple prey types: an experiental analysis of optimal foraging theory. Amer Natur $120: 297-316$

Lewontin RC (1979) Fitness survival and optimality. Pages 3-21. In: Horn HR, Stairs GR, Mitchell RD (eds), Analysis of Ecological Systems, Ohio State University Press p 312

Menge BA (1972) Foraging strategy of a starfish in relation to actual prey availability and environmental predictability. Ecol Mon 42:25-50

Menge J, Lubchenco (1974) Prey selection and foraging period of the predaceous rocky intertidal gastropod Acanthina punctulata. Oecologia (Berlin) 17:293-316

Milinski M, Heller R (1978) Influence of a predator on the optimal foraging behavior of sticklebacks (Gastrosteus aculeatus). Nature (London) 275: 642-644

Miller SL (1974) Adaptive design of locomotion and foot form in prosobranch gastropods. J Exp Mar Biol Ecol 14:99-156

Murdoch WW (1969) Switching in general predators: experients on predator specificity and stability of prey populations Ecol Mon 39:335-354

Murdoch WW, Avery S, Smyth MEB (1975) Switching in predatory fish. Ecology 56;1094-1105 
Newsome GE, Gee JH (1978) Preference and selection of prey by creek chub (Semotilus atromaculatus) inhabiting the Mink River, Manitoba. Can J Zool 56:2486-2497

Ostfeld RS (1982) Foraging strategies and prey switching in the California sea otter. Oecologia (Berlin) 53:170-178

Palmer AR (1980) A comparative and experimental study of feeding and growth in thaidid gastropods. PhD dissertation, Univ Wash, Seattle $320 \mathrm{pp}$

Palmer AR (1982) Growth in marine gastropods: a non-destructive technique for measuring shell and body weights. Malacologia $23: 63-73$

Palmer AR (1983) Growth rates as a measure of food value in thaidid gastropods: assumptions and implications for prey morphology and distribution. J exp mar Biol Ecol 73:95124

Pulliam HR (1974) On the theory of optimal diets. Amer Nat $108: 59-74$

Pulliam HR (1975) Diet optimization with nutrient constraints. Amer Natur 109:765-768

Ricketts EF, Calvin J, Hedgepeth JW (1968) Between Pacific Tides. Stanford Univ Pr, Stanford, $614 \mathrm{pp}$

Royama T (1970) Factors governing the hunting behavior and selection of food by the great tit Parus major. J Anim Ecol $39: 619-668$
Schluter D (1981) Does the theory of optimal diets apply in complex environments? Amer Natur 118:139-147

Schoener TW (1971) The theory of feeding strategies. Ann Rev Ecol Syst 2:369-404

Sih A (1980) Optimal behavior: can foragers balance two competing demands? Science 210:1041-1043

Sih A (1982) Foraging strategies and the avoidance of predation by an aquatic insect, Notonecta hoffmanni. Ecology 63:786-798

Snyder NFR, Snyder HA (1971) Pheromone-mediated behavior of Fasciolaris tulipa. Anim Behav 19:257-268

Werner EE, Hall DJ (1974) Optimal foraging and the size selection of prey by the bluegill sunfish, (Lepamis macrochirus). Ecology $55: 1042-1052$

Werner EE, Mittlebach GG (1981) Optimal foraging: field tests of optimal diet choice and habitat switching. Amer Zool 21:813-829

Wood L (1968) Physiological and ecological aspects of prey selection by the marine gastropod Urosalpinx cinerea (Prosobranchia: Muricidae). Malacologia 6:267-320

Zach R (1978) Selection and dropping of whelks by northwestern crows. Behavior 67:134-147

Received October 20, 1983 